\section{Reply to Jane}

Journal of the Operational Research Society (2007) 58, 1395. doi:10.1057/palgrave.jors.2602460

My method proposed in the published paper entitled 'A Novel Method for the Network Reliability in Terms of Capacitated-Minimum-Paths without Knowing Minimum-Paths in Advance' (J Opl Res Soc, 2005, Vol. 56, pp 1235-1240) can be implemented to search for all CMPs in the network of which the state (ie the capacity level) of each arc, for example arc $e$, is $0,1,2, \ldots, W(e)$.

If the arc state is defined as in the special cases discussed in Jane's comment, two modifications are needed for the proposed method to find all CMPs as follows:

1. The state variable $x_{i}$ (in the definition of $X$ of Section 2 and in Equation (9) of Theorem 2) needs to change from the original definition ' $x_{i}=0,1, \ldots, W\left(e_{i}\right)$ ' to the new definition ' $x_{i} \in\left\{c_{i k} \mid\right.$ where $c_{i k}$ is the $k$ th state of $\left.e_{i} \in E\right\}$ '.

2. Change Step 1 in the proposed algorithm of Section 4: Step 1 (Implement Theorem 2): Construct and use the implicit algorithm to solve all of the feasible solutions (CMP candidates), say $p_{1}, p_{2}, \ldots, p_{\pi}$, to Equations (7)-(9). If there is any feasible solution, go to Step 2. If no feasible solution exists and $d$ is less than the max-flow in the network, let $d=d+1$ and go to Step 1. Otherwise, halt.

After the above two simple modifications, all CMPs can be found using my method without needing any MPs in advance.

National Tsing Hua University

W-C Yeh

\section{Comment on Berman and Huang (2004): Minisum collection depots location problem reduces to the $p$-median problem}

Journal of the Operational Research Society (2007) 58, 1395-1396. doi:10.1057/palgrave.jors.2602433

Berman and Huang (2004) introduce the minisum collection depots location problem with multiple facilities on a network. In this note, we show that the problem is equivalent to the $p$-median problem. Let $G=(N, L)$ be a graph where the node set $N$ denotes $n$ customers and the edge set $L$ represents the links among them. $X=\left\{x_{1}, x_{2}, \ldots, x_{m}\right\}$ is the set of $m$ collection depots on $G$, not necessarily on the nodes only. $\omega_{i}$ is the weight of customer $i$ and $d(u, v)$ is the shortest distance between any points $u$ and $v$ on $G$. Given the locations of customers and collection depots, the problem is to locate $p$ facilities to minimize the total (weighted) routing distances, where each route is devoted to a single customer and involves three stops for servicing. A route originates from a starting facility, moves to a customer for picking up the material at its location, delivers the material to a collection depot, and returns to the starting facility.

Berman and Huang prove that an optimal set of facility locations of the problem belongs to an extended node set $N^{\prime}=N \cup X$. They propose the following mixed integer programming model to formulate the problem. Let

$$
z_{i j k}=\left\{\begin{array}{cc}
1 & \begin{array}{r}
\text { if node } i \text { is assigned to the facility at } j \text { and } \\
\text { depot } k \text { is selected }
\end{array} \\
0 & \text { otherwise }
\end{array}\right.
$$

and

$$
\pi_{j}= \begin{cases}1 & \text { if there is a facility located at } j \in N^{\prime} \\ 0 & \text { otherwise }\end{cases}
$$

Model P

$$
\min \sum_{i \in N} \sum_{j \in N^{\prime}} \sum_{k \in X} \omega_{i}[d(j, i)+d(i, k)+d(k, j)] z_{i j k}
$$

$s t$

$$
\begin{gathered}
\sum_{j \in N^{\prime}} \pi_{j}=p \\
\sum_{j \in N^{\prime}} \sum_{k \in X} z_{i j k}=1, \quad i \in N \\
z_{i j k} \leqslant \pi_{j}, \quad i \in N, \quad j \in N^{\prime}, \quad k \in X \\
z_{i j k}, \pi_{j} \in\{0,1\}, \quad i \in N, \quad j \in N^{\prime}, \quad k \in X
\end{gathered}
$$

Model $P$ locates $p$ facilities and assigns each customer $i$ to a pair of facility $j$ and depot $k$. Note that, however, if customer $i$ is to be assigned to facility $j$, the best collection depot assignment $k^{*}$ for the $(i, j)$ pair can be selected in advance by considering the shortest links among them, that is, $k^{*}=$ $\operatorname{argmin}\{d(i, k)+d(k, j)\}$. Let $t(i, j)$ be the length of single customer route if customer $i$ is assigned to facility $j$ so that $t(i, j)=d(j, i)+\min _{k \in X}\{d(i, k)+d(k, j)\}$. Given all $t(i, j)$ values, the problem can be remodelled as follows:

$$
q_{i j}= \begin{cases}1 & \text { if node } i \text { is assigned to the facility at } j \\ 0 & \text { otherwise }\end{cases}
$$

and

$$
\pi_{j}= \begin{cases}1 & \text { if there is a facility located at } j \in N^{\prime} \\ 0 & \text { otherwise }\end{cases}
$$

Model P-median

$$
\begin{gathered}
\min \sum_{i \in N} \sum_{j \in N^{\prime}} \omega_{i} t(i, j) q_{i j} \\
\text { st } \sum_{j \in N^{\prime}} \pi_{j}=p \\
\sum_{j \in N^{\prime}} q_{i j}=1, \quad i \in N
\end{gathered}
$$

